\title{
Summary report on the major topics addressed at ISS 8 (8th International Symposium on Sturgeons)
}

Jörn Gessner (1) https://orcid.org/0000-0002-1675-0549, Thomas Friedrich (10 https://orcid.org/0000-0002-9881-5392, Harald Rosenthal

DOI

10.1111/jai.13866

Original publication date

28 February 2019

Document version

Accepted version

Published in

Applied Ichthyology

\section{Citation (Vancouver)}

Gessner J, Friedrich T, Rosenthal H. Summary report on the major topics addressed at ISS 8 (8th International Symposium on Sturgeons). Journal of Applied Ichthyology. 2019;35(1):4-10. 
1 Summary Report on the Major Topics Addressed at ISS 8 ( $8^{\text {th }}$ International

2 Symposium on Sturgeons)

3 Compiled by Jörn Gessner ${ }^{(1)}$ and Thomas Friedrich ${ }^{(2)}$

4 Edited by Harald Rosenthal ${ }^{(3)}$ and Jörn Gessner ${ }^{(1)}$

(1) Jörn Gessner, Dept Biology and Ecology of Fish, Ecophysiology and Aquaculture; Leibniz-Institute for Freshwater Ecology and Inland Fisheries, Müggelseedamm 310, 12587 Berlin, Germany

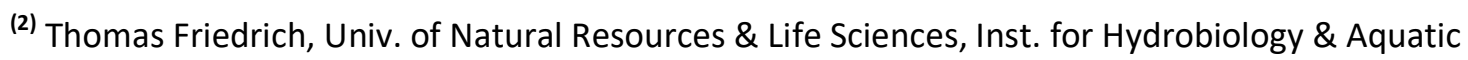
Ecosystem Management, Barawitzkagasse 8, 1190 Vienna, Austria

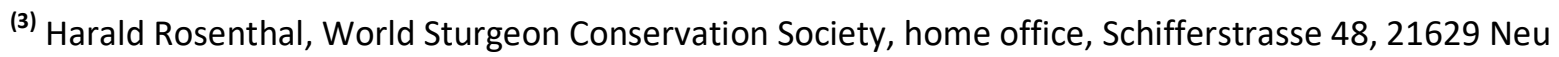
Wulmstorf, Germany

With contributions from Marie-Laure Acolas (France), Stephania Bolden (USA), Paolo Bronzi (Italy), Mikhail Chebanov (Russian Federation), Leo Congiu (Italy), James Crossmann (Canada), Jörn Gessner (Germany), Thomas Friedrich (Austria), Gertrud Haidvogl (Austria), Tim Haxton (Canada), Peter Hufschmid (Switzerland), Arne Ludwig (Germany), Florian Nagel (Denmark), Mohammad Pourkazemi (Iran), Ralf Reinartz (Germany), Harald Rosenthal (Germany) Stefan Schmutz (Austria), Andrea Schreier-Drauch (USA), Molly Webb (USA), Wei Qiwei (China), and Patrick Williot (France)

The International Symposia on Sturgeons (ISS) are held every four years, bringing together not only scientists working on sturgeons and paddlefishes around the world, but also environmental managers and experts heavily involved in nature conservation and species recovery. ISS started with its first meeting in 1989 to establish a leading global platform for sturgeon scientific exchange. This first international meeting was held in Bordeaux, France (October 3-6, 1989) followed by ISS2 in Moscow, Russia (September 5-11, 1993). Subsequently, the ISS took place in Piacenza, Italy (ISS3, July 8-11, 1997), moving to Oshkosh, Wisconsin, USA (ISS4, July 8 - 13, 2001), to Ramsar, Iran (ISS 5; May 25-31, 2005) and Wuhan, China (ISS 6; October 25-31, 2009). In 2013, the Vancouver Island University, Nanaimo, Canada, organized the $7^{\text {th }}$ ISS, (21-25 July). 
Certainly, all of these Symposia included global aspects but also had a regional focus, allowing the regional/local experts to arrange for intensified scientific exchange contributing to regional issues with an international perspective.

The $8^{\text {th }}$ International Symposium on Sturgeons, Vienna September 10-15 2017 focused on a wide range of issues with emphasis on status and management of sturgeon populations as well as aquaculture. About 320 participants from 30 countries attended the meeting. While the subject areas "General Biology" and "Ecology" received less attention than in previous Sturgeon Symposia (also with regard to the number of presentations), the main focus shifted to conservation related topics, reflecting the need to more urgently concentrate on saving the few remaining but rapidly dwindling sturgeon populations in many parts of their natural range. This was also stimulated by the focal (regional) themes of the meeting, obtaining substantial inputs from non-governmental organizations (NGO's) such as the World Wildlife Fund (WWF).

Here, we provide a brief overview on the main subject areas covered by the Conference and highlight some of the issues which were addressed in detail. Manuscripts from oral and poster presentations have been collected and submitted to a peer review process. Papers that passed the evaluation process are published in the present volume which is a special issue of the Journal of Applied Ichthyology.

The Symposium covered four major topics (General Ecology, Human Impacts, Status \& Management of Populations, Aquaculture) with 16 subtopics addressing a wide variety of issues from basic ecological questions to management related subjects. Naturally, a considerable overlap was observed between several interlinked subject areas with various contributions approaching the issues from different perspectives. In general it was noticed that genetic aspects increasingly gained importance in sturgeon research, being applied in different contexts. Out of six presentations in the first session (contributed by scientists from five different countries) topics covered ranged from the evolution of polyploidy (Vasil'yeva et al., Flajshans), to population genetics (Congiu, Mugue, Fazekas), and ecophenotypic variation (Bani). The subsequent session on genetics addressed questions on improved methods for the genetic 
identification of management units (Welsh, Congiu), phylogeny and zoogeography (Mugue), as well as the quantification of genetic diversity both in captive and wild stocks of $\mathrm{H}$. dauricus (Zhang). The overall approach towards conservation of sturgeon biodiversity was challenged in a presentation attempting to outline criteria for prioritization of conservation objectives (Cheng).

One major challenge in the field of sturgeon Genetics research became clear: Advances could be more rapidly achieved, if greater international collaboration would be more vigorously supported. Scientists working in similar areas could benefit from sharing samples or facilities to improve the overall quality and comparability of research results. Students studying genetics could increase their competency by visiting the labs of established researchers. It was decided to address this need for greater collaboration by establishing a cooperative worldwide network among sturgeon geneticists. The World Sturgeon Conservation Society's "Genetics and Genomics Working Group" was formed to take a leading role to develop this collaborative network.

Under the topic of General Ecology, the session "Reproductive Physiology" focused on (i) reproductive tools for both, field and laboratory applications, (ii) advances in assignment of sex and stage of maturity, (iii) precise identification of reproductive mechanisms in captive populations, and (iv) describing functional aspects of the reproductive systems in sturgeons. Webb et al. discussed assignment of sex and stage of maturity of hatchery-origin fish in the Columbia River with the objective of estimating the proportion of the hatchery population that are contributing to natural spawning. Results from their work are important to assess the longterm genetic risks associated with programs on the recovery of wild populations. Hu et al. evaluated controlled spawning of intersex sterlet sturgeons and evaluated the gene expression during sexual differentiation. The authors successfully induced ovulation and spermiation of intersex fish and two self-bred lines were developed for further genetic analysis. Understanding how differential genes are expressed during reproduction can improve our understanding of the mechanisms controlling sexual differentiation. Zhang et al. performed quantitative proteomic analysis of testis and ovary of the Amur sturgeon to identify proteins involved in reproduction and sex determination. They were successful in identifying some metabolic pathways potentially involved during reproduction that may advance cell development, protect the immune system, 
and promote protein synthesis. Gilroy and Litvak demonstrated how to quantify sperm quality and quantity from hatchery and wild-origin shortnose sturgeon. Their results help improve our understanding of the effects of rearing conditions on spermatozoa kinematics and seminal plasma characteristics. Further, their study provided ideas for additional research that could help optimizing conditions for controlled fertilization in aquaculture and describe reproductive behavior of the fish in the wild.

The General Ecology session comprised four contributions that dealt with applied approaches towards ecology. Specifically, the subjects focused on: (i) expert knowledge and beliefs, (ii) overwintering aggregations of shortnose sturgeon, (iii) modeling of downstream migration of juvenile European sturgeon and (iv) development of stress response in the lake sturgeon. Certainly, more research of this nature is needed to allow a more comprehensive practical application of these conceptual approaches in sturgeon conservation but it was generally felt that the outcome of such studies can provide promising components to develop management strategies.

The session "Status \& Management of Populations" received a high number of contributions in several sub-topics. The first sub-session on "Restoration Ecology" comprised eight presentations (Sherman Collier, Bronzi et al., Breve et al., Guchmanidze et al., Memis et al., Arndt et al., Friedrich et al., and Gessner et al.), providing an initial overview on current and ongoing restoration projects in different watersheds from the US to Eurasia, identifying their different foci. The main topics addressed and highlighted in the subsequent question/discussion periods noted the importance of collaborative and interdisciplinary work needed for (i) conducting successful management measures and effective future project developments; (ii) accepting the necessity to assure upfront the availability of genetically suitable animals for reintroductions; (iii) accepting the significance to prioritize research to gain detailed knowledge on the populations, their life-cycle, and habitat utilization (in order to design appropriate restoration measures and projects); (iv) recognize the absolute necessity to involve the public in planned restoration measures, as well as the need to ensure a quick and properly targeted dissemination of the issue through all political and administrative levels. It should also be understood that sturgeons offer 
118 the unique benefit in communicating strategies that are also relevant for conservation of other

119 species, including their specific habitats. Therefore, the importance of sturgeons as "flagship

120 species" was re-iterated as has been increasingly pointed out in past Sturgeon Symposia.

121 Sturgeons exhibit also a particular appeal for public attention which makes them more attractive

122 for communication purposes than most other fish species.

123 The session entitled "Habitat Assessment" included presentations with a focus on (i) dispersal of

124 young-of-the-year (YOY) following release, (ii) the use of telemetry to identify habitat utility and

125 movements within and between habitats, and (iii) combining hydro-acoustics and telemetry to

126 estimate abundance and habitat use. Breve et al. conducted tracking studies on late juvenile

127 European sturgeon to assess their survival and to determine their downstream migration in the

128 Rhine River. Similar to Breve, Acolas et al. tracked European sturgeon juveniles that were

129 released in the Gironde-Garonne-Dordogne Basin at different life phases to assess their potential

130 contribution to the wild population. O'Connor et al. combined population assessments with

131 telemetry to determine lake sturgeon habitat use in a large study area. Wu et al. also used

132 telemetry data and recapture information to study the seaward migration behavior and

133 distribution of released second generation cultured juvenile Chinese sturgeon. Knowledge on the

134 migration and distribution patterns of the stocked juvenile Chinese sturgeon will help to establish

135 an effective protection strategy for the species in the Yangtze River. Fox et al. used a novel

136 approach to infer abundance in habitats by combining acoustic receiver networks with high-

137 resolution side-scan sonar to provide the first direct estimates of the population size of the

138 annual spawning run in the Hudson River. This method has the potential to become important

139 for listed species where conventional methods to assess abundance are difficult to apply. An

140 overall conclusion was that in telemetry benefits arise from continued collaboration between

141 researchers to align methodologies and, in particular, harmonize data analyses. This is an issue

142 particularly in areas where cross-country cooperation is needed for sturgeons migrating through

143 shared watersheds.

144 The "Population Assessment" session received presentations on (i) estimating abundance of 145 specific life cycle stages, ii) developing population models, and (iii) monitoring demographics of 146 populations, including those that are commercially exploited. In their contribution Dion and 
147 D'Amours estimated the abundance of juvenile lake sturgeon before and after a river diversion

148 project with altered flows. The authors demonstrated the use of feeding habitats in the Rupert

149 River as well as the presence of juveniles born after the river diversion in the catches. A

150

151 combination of methods including telemetry, genetics, and population assessment tools was conducted to evaluate the status of sterlet sturgeon in the Danube system (Ratschan and Zauner) resulting a lower population estimate compared to previous estimates but indicating the need to further characterize the threats and critical habitats for this population. Jarić and Gessner developed a set of age-structured population models to demonstrate the difficulties in managing long-lived species such as Atlantic sturgeon. Results from their work indicated a need for longer time horizons considered for effective management of species like sturgeon, where life history and long lifespans complicate recovery efforts. An example on the Chinese sturgeon population in the Yangtze River by Wei et al. concluded that as the natural spawning activity of the fish has ceased in recent years, currently being on the edge of extinction which renders the incorporation of ecological criteria for the operation of reservoirs an essential prerequisite. Thornton et al. presented their findings on the population assessment of shovelnose sturgeon showing that despite commercial harvest, the demographics of the population remained relatively stable. However, recent data suggest a decline in physiological condition and makes further monitoring necessary to advise management to maintain a sustainable population while at the same time supporting continued sport and commercial fishing. Finally, Haxton and Friday presented a timely discussion on the importance of the effects that is resulting from ignoring assumptions on population estimates for sturgeons. The inherent nuances associated with sturgeon behavior can lead to increased error around population estimates and should be accounted for in the design of programs and in the analysis of collected data. These warnings and suggestions were later aligned with the Stock Assessment Workshop which focused on the importance of incorporating uncertainty into population assessments.

The joint session between "Status \& Management of Populations" and "Aquaculture" focused on Broodstock Management and addressed the importance of live gene banks for ex situ sturgeon conservation. The joint session re-iterated the necessity to use only genetically suitable (fully identified as part of a founder population) and adapted animals for reintroductions. While 
the size and diversity of the captive broodstock has to be sufficient to achieve the desired restoration effects while reflecting the diversity of natural populations. Furthermore, the need for different systems of holding/ keeping and husbandry for such ex situ stocks was highlighted in order to ensure appropriate husbandry for different species, forms and stages. One obvious shortcoming was very strongly emphasized: the short time-horizons of funding programs were criticized because of their inadequacy for the long-term character of ex situ measures in species of such extended longevity. This issue has already been addressed in the RAMSAR DECLARATION on global sturgeon conservation in 2005 and has now been reiterated in the VIENNA DECLARATION on global sturgeon conservation which was introduced at the closing session of ISS8 with the final version is appended to this summary report. In conclusion it was urged longterm commitments by granting agencies must be more forcefully addressed globally.

Great attention was paid to the conservation measures required as part of ex situ strategies, including (i) genetic conservation in Iran and Hungary (Pourkazemi, Kovacs), (ii) experiments to ensure spontaneous spawning of the sturgeon under controlled conditions (Du), and (iii) assessment of the effectiveness of various markers for conservation and genetic monitoring exemplified in European sturgeon (Roques). The relevance of all these reports underlines the need for the development of these important research areas.

From the many conference contributions, several take-home messages can be highlighted for broodstock maintenance. Different approaches should be taken to protect the genetic integrity of sturgeon broodstocks. Genetic integrity includes attention to the ploidy level and genetic diversity (Schreier, vanEenennaam, Fiske). To protect a species ploidy level, broodstocks should be screened before spawning to prevent production of spontaneous polyploidy individuals because they are "evolutionary dead-ends". Genetic diversity of F1 generation can be promoted by allowing for natural mate choice, which may become a viable strategy for captive sturgeon species of smaller body size such as Acipenser dabryanus. Natural mate choice allows for sexual selection and may reduce the impact of domestication in a hatchery setting. Additional experiments should seek to improve natural spawning conditions to increase fertilization rates. In larger species, developing new genetic markers that are powerful enough to delineate family structure in released progeny of related broodstocks will allow conservation aquaculture 
programs of critically endangered species to avoid inbreeding. Next generation sequencing techniques should be applied to develop SNP markers for broodstock management of tetraploid species.

Furthermore, the joint sessions on Broodstock Management, the ex situ and in situ strategies led also to consideration on "juvenile rearing \& fitness for release". A persisting interest in innovative systems for rearing and release of sturgeon larvae and juveniles (Florian et al.) was noted both in presentations and discussions, including the impact of environmental factors on the development of young sturgeons and the selection for fitness related traits for release into the wild (Shivaramu et al., Kazemi). The latter subject - although highly important - was underrepresented during the conference considering the interest that it generated.

The Aquaculture sessions on (i) reproduction techniques and rearing, (ii) fish diseases, (iii) nutrition and (iv) processing and marketing. More specific presentations illustrated the establishment of species in the farming sector in Europe (Williot), the production development in China (Wei et al.), and Iran (Abdolhay et al.). One main focus was related to biotechnological advances in germ cell handling and processing, as well as production of cell lines and polyploids (Lebeda, Vasilyeva). From a caviar production perspective, the development of clonal female populations received increasing interest. Also, the sex determination of sturgeons in the germline and early development phases (Yarmohammadi et al., Yue et al.) reflected a strong research focus during the last years.

Despite the importance of disease distribution and the impact both on wild populations and farmed sturgeon, only two papers (from China) were presented in ISS8. In both papers some data and analysis were presented on Antivirus related TIM genes in Acipenser dabryanus using light and electronic microscopy on the peripheral blood cells. It is highly recommended to carry out further studies in this area and place a research focus on sturgeon diseases both with a scope on aquaculture development, and the impact of climate change on natural populations and their ecosystems.

The sessions on "Nutrition" centered around the question on how feed can be improved in order to increase wellbeing, growth, as well as ovarian development (Ramezanpour, Kermat- 
233 Amirkolaie, Mohseni, Khara, Nagel, Liu, Zelaty, Korentovich, Agh, Houman). The general question

234 on how to adapt formulated sturgeon diets to the diverging requirements of different species

235 and their ontogentic stages still remains untouched. A field study attempted to determine the

236 nutritional sources using stable isotopes in order to improve knowledge on the overall feed base

237 optimum in aquaculture (Li).

238 The "Processing and Marketing" session provided insights into the diversity of methods currently

239 used in sturgeon production and market development. The trends were highlighted in more

240 depth in the afternoon during the forum on this topic, starting with an updated overview

241 presented by Bronzi et al. on the global meat and caviar productions, outlining also the

242 difficulties to obtain reliable data for this industry. The forum was moderated by Peter Hufschmid

243 who led through a series of open questions which resulted in a lively exchange of opinions. The

244 Discussion Forum resulted in an expression of interest by the producers to develop their own

245 association to address the specific and burning issues in a harmonized and united approach while

246 keeping a close cross-link with WSCS. Justin Henry will take the role as contact and coordinator.

247 The presentations held under the general topic "Human Impacts" in the sessions on "Fisheries

248 Assessment and Management" as well as "Public Dissemination and Capacity Building" started

249 with an outline of the stocking program for $A$. transmontanus in the Upper Columbia River,

250 British Columbia (Canada) to rehabilitate a non-recruiting population (Crossman et al.). The study

251 spanned a 15 year period and concluded that juvenile survival depended on stocking size while

252 genetic analysis suggested that survival was driven by maternal groups. In a presentation on

253 commercial harvest of the Saint John River population of $A$. oxyrinchus both harvest numbers and

254 population estimates were presented (Ceapa et al.) where the discussion linked back to the

255 presentation of Haxton and Friday. A project trying to change the attitude of artisans on fish

256 protection and management in the Lower Danube River showed that during the study period,

257 attitudes changed but a heavy reliance on river fish as source of income and food persisted (Jahrl

258 et al.). The study demonstrated that the government's sturgeon fishing ban left many fishermen

259 without any means for income, which in turn lead to substantial poaching. A regional overview

260 on the protection of sturgeon species in Azerabaijan postulated that all five sturgeon species will

261 be extinct within a five year period. Poaching, as well as dam management and hydro- 
morphological alterations are considered the major drivers. The survey was initially proposed to be a two year project, but revealed the necessity for a long-term commitment (Moore).

"Cultural Heritage" was addressed with case studies from the Austro-Hungarian Danube in comparison with the current public knowledge on sturgeons in the area.

\section{The sessions on "Habitat Degradation \& Adverse Impacts" as well as "Continuum and} Restoration of Migration Routes" documented that sturgeon populations are severely affected by multiple impacts such as migration barriers, water diversion, navigation, toxic materials in both water and sediments, change of environmental conditions in the sea, poaching, non-native sturgeons escaping from aquaculture facilities, etc. The studies presented provided detailed insights into the behavior and ecology of sturgeons. Examples from the development studies with sturgeon embryos show no acute (lethal) effects but sub-lethal impairments that are simply overlooked or often neglected. Also, the contributions illustrated the importance of functional mitigation measures at obstacles to allow the completion of the life cycle. The increased susceptibility of sturgeons that attempt passage in blocked river sections was shown to lead to an excessive vulnerability towards poaching (Suciu et al.). The necessary linkage of up- and downstream migration for functional and healthy populations, shown in an example from the US (Bruch et al.), is only slowly entering the management approaches. Longitudinal and latitudinal connectivity are key-issues that -along with habitat functionality -need to be much more stringently addressed in future conservation programs (Tritthart et al.). An example of the ignorance towards this key issue was given in a case study on the Danube (Badlita et al.). However, some case studies show that in spite of stocking, natural reproducing populations cannot be maintained. In the future it will be essential that aquaculture and conservation objectives should be jointly developed in order to reduce potential conflicts such as non-native escapements into the wild but also implement more vigorously the concept of culture for fitness for survival.

During the closing session, the draft VIENNA DECLARATION on Global Sturgeon Conservation" was presented. During the preparatory phase of ISS8 the Board of Directors of WSCS and members of the Scientific Program Committee felt the need that an update and expansion of the 
290 RAMSAR DECLARATION published in 2005 in conjunction with the proceedings of ISS5 is urgently 291 needed and the outcome of ISS8 as summarized above clearly indicated the validity of this need.

292 After the presentation during the closing sessions numerous suggestions from ISS8 participants 293 were communicated by correspondence. The final version of the VIENNA DECLARATION was 294 agreed upon and supported by contributors from 17 participating countries. The short version of 295 the declaration is attached as Appendix 1. The full version can be downloaded under 296 http://www.wscs.info/Vienna_Declaration_long 


\section{Appendix I}

\section{VIENNA DECLARATION on Global Sturgeon Conservation}

\section{Preamble}

Sturgeons and Paddlefishes (Order Acipenseriformes) comprise 27 species of which the vast majority are endangered (see IUCN Red List), while several of these species have reached critical status. This situation calls for more coordinated and concise action to prevent the species from extinction while at the same time sturgeons in this context also serve as umbrella species for other faunal elements that are affected by the same drivers. Sturgeons are excellent ambassadors for habitat related conservation approaches due to the fact that they utilize various habitats during the completion of their life-cycle. A consequent protection of habitats for sturgeons will evoke restrictions on the utilization of rivers, coastal and marine waters and the resources associated to them but these will benefit all faunal elements.

The World Sturgeon Conservation Society (WSCS) published the RAMSAR DECLARATION ON GLOBAL STURGEON CONSERVATION in 2005 outlining the guiding principles for measures urgently needed to ensure the future of sturgeons. To meet the persisting and emerging challenges in sturgeon conservation, the 8th International Symposium on Sturgeons (ISS 8) held from September 10th to 16th, 2017 in Vienna with a participation of about 300 sturgeon specialists from 32 countries felt the need to re-iterate, update and partly revise the key recommendations affecting the effectiveness of conservation management while at the same time emphasizing the requirement to apply best practice when planning or implementing activities to:

1) protect and preserve sturgeon species as emblematic flagship or umbrella species on biodiversity conservation for future generations;

2) account for the need for long-term and adequately-resourced sturgeon conservation measures supported by improved governance frameworks;

3) protect and restore rivers as dynamic, disturbance driven systems. Their hydro morphology and ecosystem services depend upon intact functionality as well as longitudinal/lateral connectivity;

4) ensure that attempts to restore and protect sturgeon populations to effective control and combat fraud and illegal, unregulated and unrecorded catch and trade while at the same time supporting sustainable aquaculture as an alternative mode of production of sturgeon commodities.

To serve these targets aiming at increased effectiveness of conservation and restoration of sturgeons, the ISS 8 developed the following recommendations for consideration by the respective sturgeon range states, regional and international agencies dealing with species conservation.

On behalf of the WSCS Board of Directors, of WWF International, the ISS8 Scientific Advisory Committee as well as the ISS8 participants 


\title{
Recommendations
}

\author{
Habitat Quality and Restoration \\ Habitat protection, river regulation, flood protection, and inland navigation
}

Recommendation 1: Recovery measures through ex situ conservation and re-stocking programs require urgent and vigorous in situ protection and habitat restoration measures: (a) all spawning habitats of Acipenseriformes must be identified and effectively protected through national and eventually international legislation; (b) the legal frameworks such as the EU Habitats Directive as well as Conventions such as RAMSAR, Biological Diversity, Bern, Oslo-Paris, Helsinki, Bucharest, and Barcelona must be fully implemented to effectively enhance the conservation status of the species through improvements of their different habitats.

Recommendation 2: Flood protection and inland navigation infrastructure have to be planned in an integrated fashion aiming to maintain to the greatest extent possible the natural hydrodynamics as well as to ensure connectivity and functionality of ecosystems. Infrastructure projects that have not been designed in such an integrated fashion must not be implemented.

\section{Damming}

Recommendation 3: New dams on sturgeon and paddlefish rivers should not be constructed. However, if unavoidable, they must be designed with state-of-the-art mitigation measures, such as properly designed passage facilities accommodating free upstream and downstream migration of all life phases of sturgeons (adults up to several meters long as well as fragile early life phases), other faunal elements, as well as permit sediment transport. Furthermore, these measures must also protect habitats and benthic communities in the upstream and downstream sections. Design considerations must incorporate climate change effects, anticipating the dynamic changes in precipitation patterns (e.g. extreme floods and extreme droughts) over a time horizon of at least 50 to 80 years, further reducing the proportion of the flow available for energy generation.

Recommendation 4: Dams have eliminated sturgeon spawning and overwintering habitats in river sections upstream or downstream of the installations. The existing facilities have to be retrofitted with structures for effective fish protection and passage both upstream and downstream (for early life phases and adults). Other dam impacts, for instance, on sediment transport and discharge (e.g. out of season peak discharge, hydropeaking, changes in temperature regime) require mitigation as well. Side channels with at least $30 \%$ of the flow under any conditions would massively reduce the impact of such facilities.

Recommendation 5: Where the construction of efficient fish passage is not viable as a result of the low capacity of the existing facilities, the removal of such facilities must be considered.

Recommendation 6: Prioritization of conservation and mitigation measures on sturgeon rivers should be applied at catchment level to maintain the ecological functions and to ensure the highest feasibility and the lowest adverse impact of technical infrastructure.

\section{Fisheries Management}

Recommendation 7: Fisheries management (e.g. planning, inspection, supervision, protection, and enforcement) and other conservation actions must be properly integrated at all levels to ensure that both aspects are adequately implemented by personnel with appropriate expertise and resources backed up by an efficient and integrated legal framework.

Recommendation 8: The legal prerequisites of fisheries regulations must reflect the dimension that poaching imposes on conservation efforts and on populations of long-lived species. Therefore, substantial fines and/or custodian sentences have to be in place. The judiciary should be adequately informed about the context and implications of such offenses to ensure that substantial penalties are imposed.

Recommendation 9: Communities that traditionally relied on sturgeon fisheries for their livelihood have to be supported in generating alternative means of income in order to facilitate compliance with fisheries bans or harvest slots. 
Recommendation 10: Fisheries researchers and managers are advised to rapidly develop and implement more selective harvesting methods, thereby preventing (or greatly reducing) the by-catch of sturgeons in fisheries for other target species.

\section{Species Survival and Repositories}

Recommendation 11: Preparation of activities to preserve the diversity of sturgeon populations outside of their natural habitat (ex situ conservation to save the remaining genetic heterogeneity and to develop potential brood stocks for sturgeon species that are on the brink of extinction) must receive priority and timely support in sturgeon rehabilitation programs.

Recommendation 12: Effective organization of ex situ stocks must be shared between range countries to reflect the joint responsibility for population management. This will also help to distribute associated costs of restoration as well as to reduce the risk of losses due to local negative events.

\section{Restocking, Recovery and Re-introduction Actions}

Recommendation 13: Stocking as a compensation measure is considered a temporary tool to overcome adverse environmental conditions causing recruitment failure or to initiate self-sustaining populations. A management structure at the national or regional level, according to the species distribution, must be established to coordinate the actions and standardize methods for reproduction, rearing and release.

\section{Trade Control}

Recommendation 14: Due to the detrimental impact of uncontrolled, illegal fishing on natural populations, the illegal trade in caviar, sturgeon meat and other products from sturgeons must be a focal area of enforcement actions both nationally, regionally, and internationally. Therefore, it has to include the provision of sufficient resources (including manpower, equipment, operational costs, etc.) for all relevant law enforcement agencies to effectively prevent sturgeon species from over-exploitation.

Recommendation 15: Responsible national authorities (e.g. CITES scientific \& management authorities, customs, food inspections, law enforcement agencies) are requested to establish formal/informal inter-agency groups (with the involvement of scientific institutions, customs, police, and financial crime specialists) to develop common approaches and harmonized means of tackling illegal wildlife trade, supporting each other in the respective activities and backing up competencies in dealing with legal fraud.

Recommendation 16: Inspections in production and trade are to be carried out unannounced. They must use state-of-the-art techniques (e.g. DNA and isotope analysis) that are necessary to identify the species and origin and thereby guarantee effective monitoring of trade in caviar and other sturgeon commodities. This also needs to include caviar containers with CITES labels, as long as manipulations cannot be ruled out completely.

Recommendation 17: Close cross-border coordination of enforcement actions concerning illegal trade of sturgeon products is required to cope with international criminal networks.

Recommendation 18: Enforcement authorities should increase their attention to the presence and authenticity of labels in line with the latest CITES labeling systems. The professional preparation and the method of application of labels must be standardized at an improved level to prevent misuse and prevent loss of labels during packaging, transport, and storage. Also, the printing quality of the CITES codes should be improved to minimize fraud.

Recommendation 19: To ensure full acceptance in court cases, analytical methods should be harmonized using appropriate scientific and laboratory standards, preferably with regular inter-calibration exercises between laboratories performing DNA and other investigatory analysis.

\section{Aquaculture}

Recommendation 20: Sturgeon species produced by aquaculture operations should be routinely monitored in line with national or regional (EU) regulations as well as with regard to environmental compatibility and product safety. To identify and prevent illegal import or laundering of illegally caught fish through aquaculture, the 
production and trade of sturgeons requires specific monitoring and control measures within the aquaculture industry. To prevent negative interaction between farmed and natural populations/species (e.g. hybridization, disease transmission, misidentification in case of by-catch), effective measures to prevent escapement from the farms should be implemented.

Recommendation 21: The aquaculture industry involved in sturgeon production is strongly encouraged to collaborate in identifying tracking approaches to support enforcement authorities in trade control actions. It is suggested to establish tissue repositories identifying captive stocks to allow a more efficient and fast commercialization of legal sturgeon products.

Recommendation 22: Commercial farms, culturing sturgeons for consumer markets, may in exceptional cases be important partners in conservation programs to bridge the time-window until the required public infrastructure for ex situ conservation is in place. Those farms may become conditionally involved and receive support for maintaining publicly owned broodstock of sturgeon species at brink of extinction if the following prerequisites are fulfilled:

1) supervision of the rearing process is carried out under the national/regional conservation authorities implementing the sturgeon recovery programs;

2) the wild fish (until F2 generation) are not owned by the farm but belong to the national or regional sturgeon recovery program, and the farmer is held fully reliable for their availability and the appropriate documentation;

3) the breeders are selected for reproduction based on prior genetic analysis and an agreed-upon breeding plan. The rearing of offspring is separated from production, implementing the recommendations on ex situ rearing;

4) utilization of the surplus production of progeny for commercial purposes must take into consideration the demand arising from restoration programs in the catchment before commercial use is permitted in a casebycase decision by the coordinating body.

\section{Policy Integration and Awareness-Raising}

Recommendation 23: Public awareness will need to be raised in order to support and push for political action towards implementation of all the above mentioned recommendations. The general public in sturgeon range countries should be made aware of the value of sturgeons to people and nature and their threat status. In particular, caviar consumers must learn how to avoid illegal products. Key stakeholders from various sectors need targeted information about sturgeon conservation. Awareness of key decision makers will have to be raised about the need for integrated policy responses and implementation of above mentioned recommendations.

This document was presented to and discussed by the participants of the 8th International Symposium on Sturgeons (ISS8) during the final Symposium session and finalized by correspondence through expert contributions.

\section{Edited by}

Harald Rosenthal, President, World Sturgeon Conservation Society Joern Gessner, Treasurer, World Sturgeon Conservation Society Paolo Bronzi, Vice President, World Sturgeon Conservation Society With contributions from

WSCS Board of Directors, WWF International, ISS8 Scientific Advisory Commicee (SAC), namely by Marie-Laure Acolas (France), Jürg Bloesch (Switzerland), Stephanie Bolden (USA), Mikhail Chebanov (Russia), Leonardo Congiu (Italy), James Crossman (Canada), Thomas Friedrich, (Austria), Gertrud Haidvogl (Austria), Tim Haxton (Canada), Jutta Jahrl, (Austria), Irene Lucius (Austria), Raimund Mair (Belgium), Coleman O'Criodain (Kenia), Mohammad Pourkazemi (Iran), Ralf Reinartz (Germany), Stefan Schmutz, (Austria), Andrea Schreier (USA), Beate Striebel (Austria), Ekaterina Voynova (Bulgaria), Molly H. Webb (USA), Qiwei Wei (China), Amy Welsh (USA), Patrick Williot (France), and ISS 8 partcipants during the Discussion Forum \& Closing sessions. 